\title{
La bioprospección: indicador de los vacíos jurídicos en los conocimientos tradicionales agrícolas
}

\author{
Bioprospection: indicator of legal vacancies of traditional agricultural knowledge
}

\author{
Natalia Narváez Torres \\ Pontificia Universidad Javeriana; Facultad de Estudios Ambientales y Rurales. Carrera de Ecología \\ Universidad del Rosario; Facultad de Jurisprudencia Especialización en Derecho Ambiental \\ Correo electrónico: narvaeznatalia05@gmail.com
}

Fecha de recepción: noviembre 30 de 2021

Fecha de aprobación: junio 15 de 2021

Para citar este artículo / To reference this article Narváez, N. (2021) La bioprospección: indixcador de los vacíos juríducos en los conocimientos tradicionales agrícolas. Inciso, 23(1) e:1109

DOI: http://dx.doi.org/10.18634/incj.23v.1i.1109

\section{Resumen}

En busca de un desarrollo sostenible. en equilibrio entre lo económico- ecológico- cultural, la bioprospección ha tenido gran importancia como estrategia integral para los países en desarrollo. Colombia, país megadiverso, podría ser líder en estos proyectos, pero al abordar el marco normativo que rige al país para el acceso de los recursos genéticos, el reconocimiento de los conocimientos tradicionales y el régimen de propiedad intelectual, se encuentran vacíos jurídicos que evitan el adecuado desarrollo de esta actividad, al no garantizar la división justa y equitativa de los beneficios obtenidos, la conservación de los recursos naturales y salvaguardar los conocimientos tradicionales delos grupos etnicos.

Ante esta problemática, esta reflexión utilizó un enfoque metodológico deductivo, donde realizó una revisión bibliográfica y análisis normativo con el propósito de estudiar en primer la bioprospección, la normativa aplicable sobre aquel en el ordenamiento colombiano, y demostrar los vacíos jurídicos que resalta la bioprospección frente a la regulación de los conocimientos agrícolas. LO que llevo a demostrar que debido a la visión antrópica por la cual nos hemos 
regido la presión sobre la biodiversidad ha ocasionado impactos negativos en la naturaleza; pero desde la transición a una visión ecocéntrica se han buscado alternativas que permitan lograr un desarrollo sostenible en equilibro, como la bioprospección. Pero para alcanzar su adecuado desarrollo se determinó la necesidad que tiene Colombia de construir una política que permita rectificar la manera de sus leyes en el cambio de un derecho clásico a un derecho ambiental que comprende e integra las funciones ecológicas y sociales.

Palabras clave: administración de justicia, legislación ambiental, indicadores socioeconómicos, conocimientos tradicionales, desarrollo sostenible

\section{Abstract}

In search of sustainable development where there is a balance between economic, ecological and cultural components, bioprospecting has been of great importance as a comprehensive strategy for developing countries. Colombia, a megadiverse country, could be a leader in these projects, but when addressing the regulatory framework that governs the country for access to genetic resources, the recognition of traditional knowledge and the intellectual property regime, there are legal gaps that prevent the proper development of this activity, by not guaranteeing the fair and equitable division of the benefits obtained, the conservation of natural resources and safeguarding the traditional knowledge of ethnic groups.

Faced with this problem, this reflection used a deductive methodological approach, where he carried out a literature review and normative analysis with the purpose of first studying bioprospecting, the applicable regulations on it in the Colombian system, and demonstrating the legal gaps highlighted by bioprospecting compared to the regulation of agricultural knowledge. Which led to show that due to the anthropic vision by which we have governed the pressure on biodiversity has caused negative impacts on nature; but since the transition to an ecocentric vision, alternatives have been sought that allow achieving sustainable development in balance, such as bioprospecting. But to achieve its proper development, it was determined that Colombia needs to construct a policy that will make it possible to rectify the manner of its laws in the change from a classic right to an environmental right that includes and integrates ecological and social functions.

Keywords: administration of justice, legislation, environmental legislation, socioeconomic indicators, traditional knowledge, agriculture, sustainable development.

\section{Introducción}

Desde los inicios de la humanidad el hombre ha utilizado los recursos naturales a su favor, divulgando sus saberes de generación en generación, a lo que se le ha denominado conocimiento tradicional. El cual se puede diferenciar en aspectos culturales, medicinales, ambientales y agrícolas. Este último está enfocado a la siembra y cuidados para el crecimiento de las cosechas, aprovechamiento, preservación y obtención de diversas variedades, oportuno para la agricultura y alimentación (Vargas-Chaves, Rodríguez y Blumenkranc, 2020). 
El conocimiento tradicional agrícola, se destaca debido a la función social que desempeña, al estar relacionado con el desarrollo de las sociedades. Lo anterior, mediante el equilibrio que alcanza entre el uso de la naturaleza y su conservación (Melgarejo et al., 2002). Adicionalmente, porque ha sido la base para garantizar la autonomía alimentaria de las comunidades campesinas y pueblos indígenas, y se ha empezado a considerar como alternativa para conseguir una soberanía alimentaria a nivel mundial (Vargas-Chaves, et al. 2020).

Con el crecimiento poblacional y los intereses económicos de los Estados se han incrementado las cantidades de recursos naturales que se necesitan, aumentando así la presión sobre estos y generando una afectación en el ciclo de resiliencia, lo que ha aumentado los niveles de pérdida de biodiversidad y alterado la forma cómo las comunidades de campesinos e indígenas utilizan estos recursos para su subsistencia (Grau, 2013).

Colombia posee más de 22 millones de hectáreas con características para la producción agrícola de cultivos (Contraloría General de la Republica, 2012), de los cuales se estima que en los últimos 20 años menos del 5\% han sido aprovechadas para la siembra de semillas nativas con conocimiento tradicional (Banco Mundial y Fondo Mundial para la Reducción de Desastres y la Recuperación, 2012; FAO, 2004).

Fenómeno originado debido a la industrialización de la agricultura y la demanda de los mercados, resultando en una agricultura intensiva. Amenaza a la que se enfrentan los conocimientos tradicionales agrícolas, sumado a la prohibición del intercambio y conservación de las semillas para una posterior resiembra, por ejemplo, para el caso de Colombia con las resoluciones 970 de 2010 y 3168 de 2015, las cuales se desarrollaran más adelante en el texto.

Es importante resaltar, que estas actividades son elementos esenciales en la cultura de las comunidades campesinas y pueblos indígenas, para su soberanía y autonomía alimentaria, así como para mantener la diversidad genética de las especies cultivadas (Helfer, 2002; Hernández, 2000).

Actualmente, enmarcados con los objetivos de desarrollo sostenible (ODS), en los objetivos de desarrollo del milenio (ODM) y si se piensa que la sociedad humana necesita de los recursos naturales para su crecimiento, no es posible seguir observando la pérdida de biodiversidad como espectadores ajenos al escenario de destrucción que se presencia en el país (VargasChaves et al., 2020).

En este contexto, se hace necesario incorporar el tema de biodiversidad (Grau, 2013), con énfasis en los recursos genéticos (Melgarejo et al., 2002), las innovaciones, el conocimiento tradicional y la propiedad intelectual en las decisiones de los gobiernos (Von Lewinski, 2008; Matiz, Rodríguez y Zuluaga, 2007). A pesar de que estén bajo el marco del denominado derecho ambiental, el cual según Cafferatta (2004) es un derecho joven, y por esta razón se pueden generar numerosos conflictos desde diversas perspectivas siendo necesario la mediación de jueces. Estos se han visto en la necesidad de adquirir un conocimiento superior en temas ambientales sin tener el criterio técnico suficiente para tal fin, permitiendo 
que se presenten inexactitudes, contradicciones y vacíos dentro del marco normativo. Se demuestra la falta de eficiencia administrativa que a su vez provoca el aumento de casos de conflictos ambientales.

Lo anterior, por ejemplo, al tener como objetivo estructurar las políticas relacionadas al conocimiento tradicional agrícola, para alcanzar el equilibrio entre la conservación de los recursos naturales y la diversidad cultural. A su vez, de lograr recuperar la agrobiodiversidad, la soberanía alimentaria y lograr la participación justa y equitativa de los beneficios económicos (PNUD, 2010).

Razón por la cual, con el propósito de proteger y regular el acceso de los recursos naturales y genéticos se han elaborado tanto acuerdos internacionales y leyes nacionales, dado que Colombia, como país megadiverso, ha sido punto de atención para múltiples proyectos de extracción (Duarte y Velho, 2009).

De esta manera es importante que Colombia enmarcada en una mirada hacia la conservación y crecimiento económico considere estrategias como la bioprospección, y cómo esta puede ser desarrollada de manera adecuada y bajo un marco normativo que permita un desarrollo económico y a la vez preserve los conocimientos tradicionales de las comunidades locales e indígenas y los recursos naturales.

Pero para lograr el adecuado desarrollo de este tipo de estrategia y considerando lo expuesto es necesario, contar con personas idóneas entre lo jurídico, técnico y científico para entender la complejidad entre los intereses económicos, sociales y de conservación de los recursos naturales y conocimientos tradicionales para de esta manera resolver los conflictos ambientales y los derechos de propiedad dentro del marco de una adecuada justicia ambiental (Pino Miklavec, 2013).

Evidencia de lo anterior es que entre las soluciones emergentes para subsanar estos vacíos en Colombia, en el año 2020 se presentó ante la Cámara de representantes el ProyectoLey 047 "Por medio del cual se dictan disposiciones para la conformación de los tribunales ambientales especializados en Colombia", buscando fortalecer la legislación ambiental, logrando que las decisiones tomadas sean bajo una perspectiva judicial-ambiental.

\section{Materiales y métodos}

Para esta reflexión se utilizó un enfoque metodológico deductivo, donde se realizó una revisión normativa y bibliográfica tomando como referencias publicaciones de expertos de diferentes áreas-incluyendo técnicos, la academia y el Estado-, con el objetivo de tener una visión holística del estado del arte en el tema de la bioprospección, además, se realizó un análisis normativo en el marco de determinar si el país cuenta con las adecuadas leyes que protejan los conocimientos tradicionales agrícolas y reconozcan un reparto justo y equitativo de los beneficios obtenidos de estos, así como de los recursos genéticos.

De esta manera, esta reflexión tiene por fin el presentar el panorama actual en el que se encuentra Colombia, desde el marco internacional y nacional, respecto al proceso de 
bioprospección como una integral para el desarrollo sostenible del país y un indicador de los vacíos jurídicos para la regulación de los conocimientos tradicionales agrícolas.

Expone la importancia que tiene que un país como Colombia reconozca los conocimientos tradicionales agrícolas y respete los derechos de las comunidades campesinas y pueblos indígenas en el marco de buscar nuevas alternativas económicas que estén en armonía con los recursos naturales, tomando como ejemplo la bioprospección.

Argumenta cómo un cambio en el modelo económico o filosófico por el que nos regimos genera la necesidad de investigar nuevas alternativas económicas, las cuales actualmente buscan un desarrollo que ocasione un mínimo impacto al ambiente y su conservación a largo plazo, un desarrollo sostenible. También hace patente cómo la necesidad de proteger la naturaleza y la diversidad cultura por parte del legislador nacional e internacional representa el reto de alcanzar una estrategia integral donde ya no prime el interés de algunos pocos de manera independiente, sino que se busque una articulación en varios niveles para conseguir un reparto justo y equitativo, una opción de gana-gana entre los actores involucrados.

\section{La bioprospección}

La bioprospección es una técnica que busca, evalúa y recopila información de conocimientos tradicionales y variedades vegetales, lo que permite identificar, seleccionar y aislar componentes para el desarrollo de nuevos productos a través del uso de conocimiento científico y herramientas tecnológicas, lo anterior con el fin de lograr una comercialización en paralelo a la conservación de la fauna y flora y evitar la erosión genética (Vargas-Chaves, 2018; Carranza, 2017; Williams y Hardison, 2013).

Esta técnica, involucra el conocimiento científico y las herramientas tecnológicas por parte de los Estados, así como la participación de las comunidades y el respeto de sus derechos, por ejemplo, el reconocimiento al conocimiento tradicional (Carranza, 2017; Duarte y Velho, 2009; Matiz et al., 2007).

La bioprospección es, por lo tanto, una estrategia integral que contempla la parte ambiental, económica, política y cultural; que logra un crecimiento económico, al mismo tiempo que genera nuevos conocimientos sobre los recursos naturales y su utilización en los procesos productivos. Aumenta la calidad de vida, mediante soluciones que mejoran las condiciones de salud, de obtención de alimentos, oportunidades de aprendizaje e investigación. Rescata diferentes culturas y reconoce los derechos de las comunidades como su participación en las decisiones, el respeto y valoración de los conocimientos tradicionales (Melgarejo et al., 2002).

Desde el sector económico la bioprospección es una alternativa de gana-gana donde la biodiversidad se contempla como un doble capital: considerando su valor de cambio y su valor de uso (O'connor, 1998), enfocada a un desarrollo sostenible y atada al capital intelectual ambiental, como lo plantea Vargas (2020). Es una estrategia para conservar el bienestar humano, los servicios ecosistémicos y, por ende, la conservación per se de la naturaleza que permite, a la vez, la extracción de insumos de la misma con un beneficio económico (O'connor, 1998). 
Hoy en día, la bioprospección tiene diversas aplicaciones descubiertas por los progresos tecnológicos y científicos en temas como biotecnología, etnobotánica y biología molecular, lo que ha permitido obtener resultados como la seguridad alimentaria, los avances en el sector agrícola, la biorremediación, la biomedicina, en cosmética y en el sector industrial y tecnológico (Carranza, 2017; Duarte y Velho, 2009; Melgarejo et al., 2002). De esta manera, se ofrecen beneficios económicos intersectoriales.

Actualmente, una de las aplicaciones de la bioprospección con mayor interés a nivel global es su utilización para la soberanía alimentaria. Esto se debe, entre otros factores, al establecimiento del segundo objetivo de los objetivos de desarrollo sostenible (ODS) "Hambre Cero. Poner fin al hambre, lograr la seguridad alimentaria y la mejora de la nutrición y promover la agricultura sostenible" (PNUD, 2020).

Este objetivo reconoce que aún se evidencian numerosos casos de subalimentación crónica a nivel mundial que afectan la salud, el bienestar humano y el presupuesto de las naciones. Las principales preocupaciones que dificultan el logro de este ODS son: la pérdida de los recursos naturales, la erosión genética, la extensión de tierra infértil y el aumento en la periodicidad de intensos fenómenos meteorológicos (PNUD, 2020).

Como parte de la configuración de la biodiversidad como "el eje vivo" del medio ambiente, y a modo de interés jurídico superior -en los términos de Rodríguez y Vargas-Chaves (2016)-, y en la dirección planteada hasta ahora, es que se ha dado relevancia a la agrobiodiversidad, tal como se señaló en la consulta internacional: "Función de la agrobiodiversidad en el logro del Objetivo de Desarrollo para el Milenio de erradicar el hambre y la pobreza”. Allí, se resaltó la importancia de considerar el potencial de la agrobiodiversidad como alternativa para mejorar el bienestar de los humanos y fuente de nuevos ingresos económicos (Cotes et al., 2012).

De la misma manera como durante la Cumbre Mundial Sobre la Alimentación (1996), se declaró la propia soberanía alimentaria como el derecho que tienen todos los grupos étnicos y culturales a contar con una calidad y cantidad de alimentos suficientes para satisfacer sus necesidades y poder llevar un adecuado nivel de vida. De esta manera es declarado derecho superior, teniendo presente que mediante la soberanía alimentaria se protege el derecho a la vida. Por consiguiente, no deberían existir restricciones a la hora de utilizar el conocimiento tradicional agrícola (Mendoza-Cerquera, 2015).

Dentro de este marco, la diversidad biológica, por lo tanto, genética que tienen los países en desarrollo, con sus múltiples variedades, su capacidad de resistir a enfermedades y plagas, son de alto atractivo para los sistemas agropecuarios debido a que entre más fuertes sean las características del germoplasma, resulta mejor para los bancos genéticos (Melgarejo et al., 2002).

Variedades que se han logrado gracias a los conocimientos tradicionales que presentan las culturas, así como por la utilización que las personas que habitan los territorios han realizado de estos recursos naturales generación tras generación para su subsistencia (Sunder, 2007); por eso es tan importante reconocerles su conocimiento tradicional y la forma como han 
conservado la naturaleza (Vargas-Chaves, 2018).

No en vano el Gobierno colombiano le ha apostado, como una estrategia de desarrollo sostenible, a la creación de bancos de germoplasma para la alimentación y la agricultura. Además, por medio del Ministerio de Agricultura y Desarrollo Rural (MADR) asignó a la Corporación Colombiana de Investigación Agropecuaria (Corpoica), representada por el Centro de Biotecnología y Bioindustria (CBB), la responsabilidad de coordinar y articular la estructuración de un programa nacional en bioprospección enfocado al sector agropecuario del país.

\section{Normatividad adjudicable a la bioprospección}

A partir de la necesidad de lineamientos para la conservación de los recursos naturales y genéticos y conocimientos tradicionales que permitan un desarrollo adecuado de estrategias económicas como la bioprospección, garantizando la división justa y equitativa de los beneficios obtenidos de esta; Colombia ha planificado sus políticas y marcos normativos con base en convenios internacionales y normas internas como las mencionadas a continuación:

La Convención sobre la Diversidad Biológica (CDB): plantea la conservación y el uso sostenible de la biodiversidad y el reparto justo y equitativo de los beneficios obtenidos. Adicionalmente, la establece que antes del acceso a los recursos genéticos se deberá contar con un consentimiento previo por parte del Estado, mismo que debe proteger el conocimiento tradicional asociado, las innovaciones y prácticas de las comunidades étnicas y reconoce el derecho a participar en la toma de decisiones relacionadas con la conservación y el uso sostenible de los recursos genéticos (Ley 165, 1994).

Esta es la primera vez en que internacionalmente se destaca el vínculo que existe entre el uso y la conservación de la diversidad biológica, los conocimientos tradicionales y las nuevas creaciones. Se adopta así un concepto integral de la biodiversidad, desde el componente biológico, cultural, político y económico.

El Convenio 169 de la OIT: estipula que los Estados son responsables de gestionar espacios que permitan la participación de las comunidades indígenas; realizar consultas previas con el fin de obtener el consentimiento libre, previo e informado (CLPI), y resguardar sus conocimientos tradicionales y costumbres bajo el régimen de propiedad intelectual. Se declara que los pueblos indígenas tienen el derecho de que se les reconozca y respete sus propias prácticas culturales, costumbres y tradiciones (Ley 21, 1991).

La Declaración de las Naciones Unidas sobre los Derechos de los Pueblos Indígenas (DNUDPI): define cómo los gobiernos deben proteger los derechos de los pueblos indígenas, permitiéndoles el desarrollo de sus propias tradiciones como parte de la diversidad cultural de las naciones.

La Declaración Universal sobre la Diversidad Cultural: donde reconoce la diversidad cultural, de los diferentes grupos que constituyen el ser humano y declara que los conocimientos tradicionales son patrimonio común de la humanidad, por tanto, deben ser protegidos. 
La Decisión Andina 391 de 1996: regula el acceso a los recursos y sus derivados a fin de garantizar el cumplimiento de la CDB. Reconoce adecuadamente el conocimiento tradicional y el derecho que tienen las comunidades de decidir sobre este. Determina que los recursos genéticos son inalienables, imprescriptibles e inembargables, y legaliza las actividades de bioprospección. Destaca la conexión entre el acceso a los recursos genéticos y los derechos de propiedad intelectual. (Comunidad Andina, 1996).

La Decisión Andina 486 de 2000: constituye el régimen comunitario andino sobre propiedad industrial para Colombia y determina que para acceder a los mercados internacionales se debe contar con la procedencia legal de los recursos. Además, no considera como invención o patentable el material biológico o genético, a pesar de que haya sido manipulado (Comunidad Andina, 2003).

El Protocolo de Nagoya: reitera que es menester obtener el consentimiento libre, previo e informado (CLPI) del país propietario del recurso genético antes de acceder a este, así como negociar las condiciones del acceso, la participación de las comunidades y el desarrollo de la investigación en pro de la conservación y del uso sostenible de la naturaleza. También resalta la importancia que tienen los recursos genéticos para el sector agrícola y la seguridad alimentaria (Ley 740 de 2002).

Tratado internacional sobre recursos fitogenéticos para la alimentación y la agricultura: regula los temas relacionados con los recursos fitogenéticos destinados a la agricultura y seguridad alimentaria. Promueve las técnicas de conservación con la colaboración de las comunidades. Además, entrega los requisitos que los Estados deben cumplir para garantizar la protección de los conocimientos tradicionales (FAO, 2009).

El Protocolo de Cartagena: señala que para realizar actividades de bioprospección con mejoramiento genético se debe contar con la autorización de la autoridad competente (Ley 1926 de 2018).

El Código de los Recursos Naturales: en su artículo 291 afirma que se debe solicitar una autorización para la comercialización o importación de los productos logrados a partir de la utilización de recursos naturales o genéticos (Decreto 2811, 1974).

La Constitución Política de Colombia: en sus artículos 7, 8 y 81 manifiesta que es obligación del Estado proteger la diversidad biológica y cultural del país, por ejemplo, regulando la importación y exportación de los recursos genéticos, y el uso que se hace de estos. En sus artículos 79 y 330 establece que el Estado es el responsable de garantizar los derechos de los pueblos indígenas, por ejemplo, el derecho a la participación.

La Ley 1032 de 2006: por medio de la cual se modificó el artículo 306 del Código Penal, y obliga a los agricultores a utilizar semillas certificadas para sus cultivos.

La Ley 1453 de 2011: en su artículo 37 decreta que aquel que realice algún tipo de actividad que cause un impacto negativo en la biodiversidad o en la salud humana, sin los permisos necesarios o en incumplimiento de la normatividad, incurrirá en prisión. 
La Ley 1333 de 2009: en su artículo 5 considera infracción ambiental todas aquellas acciones $u$ omisiones de las normas y actos administrativos vigentes.

Decreto 1397 de 1996: por el cual se crea la Comisión Nacional de Territorios Indígenas y la Mesa Permanente de Concertación con los Pueblos y Organizaciones Indígenas, con el propósito de defender las prácticas y conocimientos tradicionales, conciliando con las comunidades indígenas las propuestas generadas por el Estado para la protección de sus derechos a la hora de acceder a los recursos genéticos. Asimismo, pactar la legislación ambiental y los derechos constitucionales indígenas.

El Decreto 4525 de 2005: por el cual se reglamenta la Ley 740 de 2002que permite a extranjeros introducir en Colombia organismos genéticamente modificados, incluyendo semillas.

El Decreto 1076 de 2015: en sus capítulos 8 y 9 se reglamentan los permisos de recolecciones de especies de flora y fauna para el conocimiento científico, el funcionamiento y registro de las colecciones biológicas y los derechos y deberes de los titulares de estas.

La Resolución 187 de 2006: Por la cual se adopta el Reglamento para la producción primaria, procesamiento, empacado, etiquetado, almacenamiento, certificación, importación, comercialización, y se establece el Sistema de Control de Productos Agropecuarios Ecológicos. Además, se reguló el uso de las semillas obtenidas durante la siembra y estandarizó las semillas certificadas.

La Resolución 970 de 2010: Por medio de la cual se establecen los requisitos para la producción, acondicionamiento, importación, exportación, almacenamiento, comercialización y/o uso de semillas para siembra en el país, su control y se dictan otras disposiciones.

La Resolución 1348 de 2014: a través de esta: "Se establecen las actividades que configuran acceso a los recursos genéticos y sus productos derivados para la aplicación de la Decisión Andina 391 de 1996 en Colombia y se toman otras determinaciones" (Resolución 1348 de 2014).

La Resolución 3168 de 2015: Por medio de la cual se reglamenta y controla la producción, importación y exportación de semillas producto del mejoramiento genético para la comercialización y siembra en el país, así como el registro de las unidades de evaluación agronómica y/o unidades de investigación en fitomejoramiento y se dictan otras disposiciones.

La Política para el Desarrollo Comercial de la Biotecnología a partir del Uso Sostenible de la Biodiversidad: se propone la creación de condiciones económicas, técnicas y legales que permitan conseguir beneficios públicos y privados para la comercialización de los bienes o servicios obtenidos gracias al uso sostenible de la biodiversidad. Considera diferentes herramientas que sean beneficiosas para el país, como la bioprospección (Conpes 3697, 2011). 
La política Nacional para la Gestión Integral de la Biodiversidad y sus Servicios Ecosistémicos: la cual tiene como objetivo promover la Gestión Integral de la Biodiversidad y sus Servicios Ecosistémicos, reconociendo la distribución justa y equitativa de los beneficios derivados del aprovechamiento de los conocimientos, innovaciones y practicas referentes a la conservación y uso sostenible de la naturaleza.

\section{La bioprospección como un indicador de los vacíos jurídicos de los conocimientos tradicionales agrícolas}

Desde la Declaración de las Naciones Unidas sobre los Derechos de los Pueblos Indígenas (DNUDPI), se hacen evidentes las injusticias que han experimentado los pueblos indígenas debido a que desde la época de la colonia se les han arrebatado sus tierras y recursos naturales necesarios para poder desarrollar su cultura adecuadamente.

Situación que actualmente sigue presentándose efecto de los intereses económicos de diferentes empresas, para intervenir en los territorios de las comunidades campesinas y pueblos indígenas y explotar los recursos naturales y genéticos que ahí se encuentran, generando un posible incumplimiento al derecho de estas comunidades de su libre manifestación cultural (Procuraduría General de la Nación, 2009).

De esta manera, el Tribunal constitucional (Sentencia T-769 de 2009) planteo la importancia de lograr un equilibrio entre el desarrollo económico y la explotación de los recursos naturales y genéticos del país, que además son parte esencial de la integridad de las comunidades locales.

Actualmente, en el país se evidencian varios proyectos de infraestructura, de minería, de explotación de recursos, como la bioprospección, que buscan un desarrollo económico dentro de los territorios de las comunidades campesinas y pueblos indígenas que justificados con el desarrollo nacional y bienestar de los colombianos, han avanzado de manera arbitraria afectando directamente a estas comunidades (T- 428 de 1992, SU-039 de 1997, T- 652 de 1998, SU- 383 de 2003, T-880 de 2006, C-030 de 2008, C-461 de 2008, C- 715 de 2009 y T-769 de 2009). Adicionalmente, rompiendo la función ecológica de la propiedad, la cual hace referencia a los derechos de las generaciones futuras de gozar de un ambiente sano (Constitución Política de Colombia, 1991).

Lastimosamente, es importante mencionar que no obstante a las múltiples veces que este término se ha usado en la jurisprudencia colombiana la ausencia de su definición es eminente. Lo que ocasiona que cada legislador tenga la libertad de interpretarla indistintamente, ocasionando cambios en el alcance, límites y medios de control, producidopor los vacíos jurídicos derivados del denominado derecho ambiental joven de Cafferatta (2004).

Se vuelve, por lo tanto, menester que toda persona que quiera desarrollar alternativas económicas basadas en recursos naturales necesita hacerlo desde las obligaciones que se encuentran en el articulo 95 de la Constitución Política “(...) 1. Respetar los derechos ajenos y no abusar de los propios. (...) 8. Proteger los recursos culturales y naturales del país y velar por la conservación de un ambiente sano. (...)". 
De igual manera, que subsanar este vacío en la jurisprudencia colombiana. En el marco que al definir la función ecológica de la propiedad se puede regular adecuadamente la propiedad tanto pública como privada y las condiciones que las empresas o el Estado deben cumplir cuando deseen utilizar los territorios pertenecientes a las comunidades campesinas y pueblos indígenas (Rodríguez, G. A., Gómez Rey, A., y Sáenz Quitian, A., 2014). (Rodríguez, G. A., Gómez Rey, A., y Sáenz Quitian, A., 2014). Como es el caso de alternativas de desarrollo económico como lo es la bioprospección, enmarcadas en lograr la explotación de los recursos naturales necesarios sin afectar la integridad cultural de las comunidades que habitan los territorios ni la capacidad de resiliencia que tienen los ecosistemas.

Desde otro ángulo, también es importante aludir que pese a las regulaciones mencionadas y las demás existentes, aún subsiste el riesgo de que las actividades no generen una compensación equitativa de los beneficios que se obtengan (Melgarejo et al., 2002).

Un ejemplo de lo anterior se evidencia con la firma del Tratado de libre comercio (TLC) con Estados Unidos, donde ni la Decisión 391 ni la 486 de la Comisión De La Comunidad Andina (CAN) fueron tenidas en cuenta para el marco de referencias. Esto permite que las patentes emergentes del TLC por parte de Estados Unidos no estén obligadas a reconocer los derechos de propiedad intelectual de los recursos genéticos provenientes de Colombia, ni a dar reconocimiento al conocimiento tradicional de las comunidades (Álvarez- Tafur, 2014).

También hay un conflicto entre la CDB y el Acuerdo sobre Protección de la Propiedad Intelectual (ADPIC). Según este, las patentes se podrán solicitar si suponen innovación, reproducción, industria y demuestran que son nuevas. Adicionalmente, con el fin de conseguir un comercio más fácil, le otorga a los Estados los derechos sobre la biodiversidad, reglamentando los derechos de propiedad intelectual (Borrás, 2020). Es decir, todo producto generado a partir de los recursos naturales o genéticos obtenidos de un país serán exclusividad del titular de la patente, así como los beneficios recibidos (Borrás, 2020; VargasChaves, et al. 2020). Por ejemplo, en el caso de la bioprospección y los recursos naturales que sean explotados procedentes de Colombia, pero estudiados, investigados y modificados fuera del país al momento de ser comercializados no serán considerados en el marco de una distribución justa y equitativa de los beneficios que se obtenga.

Por consiguiente, se evidencia que no se tuvieron en cuenta los acuerdos alcanzados con la $\mathrm{CDB}$, en cuanto a considerar los requerimientos de beneficios justos y equitativos entre el poseedor de la patente y el Estado dueño de los recursos, ni en el tema del reconocimiento de los conocimientos tradicionales (Borrás, 2020).

Otro obstáculo al que se enfrenta Colombia tiene relación con las resoluciones 187 de 2006, 970 de 2010 y 3168 de 2015 emitidas por el Instituto Colombiano Agropecuario, en donde se regula la utilización de semillas para la resiembra y se declara la utilización de semillas certificadas. Esta práctica pone en riesgo el conocimiento tradicional agrícola, ocasionando que su no aplicación lo lleve a la desaparición y se pierda la variedad genética de las semillas, factores que causan una gran pérdida cultural y biológica en el país (Helfer, 2002; Hernández, 2000). 
Pérdida que se ocasionara debido a la falta de normatividad existente para la protección adecuada de los conocimientos tradicionales explotados en miras a un crecimiento económico, sin tener en cuenta un reconocimiento de propiedad intelectual de las comunidades campesinas y pueblos indígenas (Acea-Valdés, 2014).

Al hacer un análisis de la parte económica, también es importante tener presente que el porcentaje de ganancia de los agricultores se ve reducido en el momento que se les obliga a comprar semillas certificadas, en lugar de poder utilizar sus propias semillas; lo que a su vez genera un problema al momento de competir con los precios en el mercado de los agricultores industriales (VargasChaves et al., 2020).

Atendiendo estas consideraciones es importante que Colombia, antes de continuar desarrollando proyectos de extracción de los recursos naturales y genéticos con otros países, aun siga reforzando el marco legal (Matiz et al., 2007; Melgarejo et al., 2002), las políticas de acceso a recursos genéticos, los derechos de propiedad intelectual (Melgarejo et al., 2002) y de conocimiento tradicional (Matiz et al., 2007), y genere coherencia normativa para la investigación, el acceso a recursos genéticos, la biotecnología y bioprospección (Melgarejo et al., 2002).

Ello, principalmente, debido a que Colombia después de ser sede para desarrollar el proyecto del PNUD, en el marco del Objetivo de Desarrollo del Milenio 7 (ODM) que busca incorporar el conocimiento tradicional en agroecosistemas y asegurar un desarrollo sostenible, dejó al descubierto la inexactitud de sus políticas (Vargas-Chaves et al., 2020).

El país debe tener claridad a la hora de definir los propietarios de los derechos sobre un recurso genético específico o de un conocimiento tradicional (Grau, 2013), antes de ser dispuestos para alternativas de desarrollo económico, como la bioprospección, para lo cual necesita tener un adecuado registro de los diferentes conocimientos tradicionales y costumbres de las comunidades de campesinos y pueblos indígenas que le facilite su protección (OMPI, 2005). Igualmente, asegurar la conservación de la biodiversidad, mediante estrategias viables que no ocasionen impactos negativos en los ecosistemas (Rodríguez y Vargas-Chaves, 2016; Wynne, 1992).

Finalmente, es necesario que se fortalezca la capacidad técnica y científica del país, pues las dificultades más grandes que se encuentran en el proceso de bioprospección es en el paso de la fase de investigación a comercialización y en la necesidad que se tiene de realizar análisis específicos en el exterior (Carranza, 2017), lo cual ocasiona, como se mencionó que la entrada de los bioproductos al país no genere el reconocimiento del conocimiento tradicional agrícola correspondiente ni se asuma la distribución justa y equitativa de estos beneficios.

Esto exige un trabajo intersectorial por parte de la academia, el Gobierno y el sector empresarial nacional e internacional, en el que haya una visión integral de toda la cadena de valor, con objetivos claros. Además, requiere disponer de las capacidades técnicas y de desarrollo adecuadas, donde los acuerdos de investigación se respeten y estén alineados con los conocimientos necesarios y el marco jurídico (Cotes et al., 2012). 
En definitiva, Colombia al desarrollar un marco normativo sólido y coherente puede seguir desarrollando la bioprospección como alternativa para conservar su biodiversidad y obtener a la vez beneficios de esta. La cual, gracias a su agrobiodiversidad, permite generar ventajas competitivas que, mediante un manejo adecuado de aspectos jurídicos, económicos, científicos, tecnológicos y comerciales, podrían sacar ventaja de la demanda regional, nacional e incluso mundial.

\section{Una propuesta de redimensionamiento de los vacíos legales para regular la bioprospección y los conocimientos tradicionales agrícolas}

Colombia, país megabiodiverso con características excepcionales para el sector agrícola (Contraloría General de la Republica, 2012) tiene la facilidad de posesionarse como líder en el desarrollo de grandes proyectos donde se utilice la bioprospección, esto en el marco del sistema de bancos de germoplasma que se ha creado, la integración de sus diferentes instituciones (centros de investigación, academia, público y empresarial) y el incremento en la capacidad de investigación (Cotes et al., 2012).

Por ejemplo, al centrarse en productos y soluciones tecnológicas para el sector agropecuario, se ha orientado a: (a) la mejora de la calidad de los alimentos mediante la búsqueda de atributos nutricionales, nutracéuticos, organolépticos superiores y funcionales, que además contribuyen al aumento de la producción con estrategias que le permitan una ventaja competitiva; (b) la inocuidad de los alimentos, control de plagas y enfermedades, reemplazando los productos químicos utilizados por productos amigables con el ambiente; (c) la solución a problemas fitosanitarios mediante el mejoramiento genético o el uso de bioproductos; (d) la transformación de los productos o de los desechos generados por la agroindustria para darle valor agregado al sector; (e) la contribución al conocimiento de los recursos genéticos de animales, vegetales y microorganismos para la generación de estrategias de conservación, mejoramiento genético y uso sostenible de los recursos naturales, (g) el apoyo al desarrollo de innovaciones (Cotes et al., 2012).

Sin embargo, para poder ser líder en otros proyectos de bioprospección es menester que Colombia:

- Cambie la idea de tener una agricultura intensiva a una que fomente la agricultura con siembra de semillas nativas o que han sido cosechadas de generación en generación gracias al conocimiento tradicional y que permite mantener la diversidad genética.

- Reconozca la importancia de rescatar y preservar los conocimientos tradicionales.

- Genere inventarios de la diversidad de sus recursos genéticos.

- Amplíe su campo de investigación a otras áreas, más allá del sector agropecuario, como la búsqueda de condiciones que mejoren la salud humana.

- Aumente los esfuerzos para formar personal altamente calificado en temas relacionadas con la biotecnología y la formación de científicos innovadores. 
- Incremente los recursos financieros destinados al estudio de la bioprospección y busque la manera en que el Estado pueda realizar una inversión en temas de tecnología, infraestructura de laboratorios, invernaderos, plantas piloto y adquisición de equipos que permitan la investigación dentro del país, sin tener la necesidad de mandar al exterior las muestras de tejidos o ADN.

- Desarrolle alternativas que permitan la reproducción industrial y la fácil comercialización de los productos, lo anterior con el objetivo de lograr que estos lleguen al mercado nacional e internacional para contribuir con el desarrollo de las regiones y a aumentar el bienestar de los colombianos.

- Elabore un marco jurídico, así como políticas de Estado claras, coherentes, estables, que sigan los lineamientos de los tratados internacionales de conservación, uso sostenible de los recursos naturales y genéticos, reconocimiento de los conocimientos tradicionales, distribución justa y equitativa de los beneficios obtenidos y de propiedad intelectual.

Este ultimo numeral, como se ha demostrado a lo largo del texto, es indispensable debido a que brinda las bases de unas leyes sólidas y congruentes, eliminando las posibles contradicciones entre las mismas y los vacíos legales que se pueden presentar para confusión y mal interpretación. Tomando Colombia la iniciativa de transformar los vacíos legales existentes, en ideas claras para el progreso de nuevas alternativas de desarrollo sostenibles como la bioprospección. De esta manera también demostrando el cambio que ha tenido el país con la forma cómo se contemplaba el derecho, resaltando la importancia de tener presente un derecho ambiental, liderado por expertos técnicos y legisladores que le aseguren al Estado la mejor toma de decisión, al tener contemplados todos los escenarios posibles de afectación.

Igualmente, desarrollar una política desde un derecho ambiental integral, orientado a partir de un componente ecológico y cultural, al contemplar la función social de las comunidades y la relevancia de la propiedad intelectual y del conocimiento tradicional, como estrategia de conservación de los recursos naturales, las variedades genéticas y la soberanía alimentaria. También, relacionada con el respeto de la retribución justa y equitativa de los beneficios derivados de la naturaleza, respetando a las comunidades campesinas y pueblos indígenas que los poseen (Matiz et al., 2007), así como la función ecológica de la propiedad (Rodríguez, G. A., Gómez Rey, A., y Sáenz Quitian, A., 2014). De esta manera se aseguraría el país de mejorar las condiciones sociales, fomentar la diversidad cultural, conservar su biodiversidad y lograr incrementar sus ingresos económicos.

Adicionalmente, Colombia al contemplar una política de esta magnitud sería pionero en el tema. Esto enmarcado a que en la actualidad no existe un marco normativo -ni a nivel internacional o nacional- que regule desde el régimen de propiedad intelectual la protección de los conocimientos tradicionales agrícolas.

Al tener en cuenta la distribución justa y equitativa de los beneficios derivados de la naturaleza, se busca avalar un desarrollo sostenible mediante el uso de la biodiversidad y destacar el valor de los conocimientos tradicionales agrícolas como alternativa de 
conservación de estos recursos y como parte de la identidad cultural de las comunidades de campesinos y pueblos indígenas (Comisión Nacional para el Conocimientos y Uso de la Biodiversidad, 2017).

En línea con la Organización Mundial de la Propiedad Intelectual (2002), los conocimientos tradicionales agrícolas, por lo tanto, deben contemplarse con un valor monetario, el cual puede ser sujeto de los servicios o productos que de este se generen o en su defecto en su contribución en el régimen de propiedad intelectual, de esta manera, reconocerse como patrimonio intangible.

Asimismo, es necesario que se mantenga un cambio de modelo económico en el que se efectúe una transformación entre un modelo capitalista frente a la naturaleza, a uno donde haya una desmercantilización de la misma ( $0^{\prime}$ Connor, 1998), a pesar de que sean vistos los recursos naturales también como recursos económicos. Esto como estrategia para solucionar los problemas de pérdida de la biodiversidad.

Expuesto lo anterior, es evidente que la distribución justa y equitativa de los beneficios obtenidos de la naturaleza no puede estar regulada por una política común, puesto que debe estar elaborada específicamente para la situación en la que se encuentra cada Estado, para lograr el adecuado reconocimiento de los actores involucrados (Posey y Dutfield, 1996).

En lo que respecta a Colombia, se debería considerar la elaboración de una política nacional que incluya las directrices para el desarrollo de la bioprospección en el país, donde estén claros los mecanismos de financiación y las estrategias de negociación entre los diferentes actores -nacionales e internacionales-, además de la salvaguarda de los conocimientos tradicionales, el involucramiento del sector empresarial, la atracción de la inversión extranjera y la garantía de la conservación de la naturaleza. De esta forma, se puede mejorar el bienestar de los colombianos con nuevas oportunidades laborales y el incremento de ingresos al país, gracias a la comercialización de los bioproductos a nivel nacional e internacional y la ganancia producida por la utilización de los recursos genéticos y sus derivados, siempre que haya un reparto justo y equitativo de estos, entre las naciones involucradas y un reconocimiento de la propiedad intelectual.

\section{Conclusiones}

El crecimiento demográfico, el desarrollo de las ciudades, el uso de la tecnología, entre otros, son factores que han generado una alta presión sobre la biodiversidad y la manera cómo se ha utilizado la naturaleza, principalmente, cuando se contemplaba desde una visión antropocéntrica. Afortunadamente, hace varias décadas ha comenzado la transición a una visión más ecocéntrica, donde el ser humano ha percibido que para lograr su crecimiento y mantener una buena calidad de vida es necesario conservar la naturaleza y realizar un uso sostenible de la misma, pensando no solo en los intereses presentes, sino también en los futuros, a lo que se le conoce como desarrollo sostenible.

Tanto a nivel internacional como nacional, se ha trabajado en buscar alternativas integrales que permitan el crecimiento económico sin ocasionar impactos negativos significativos en los 
recursos naturales y mediante el desarrollo de marcos jurídicos dirigidos a la protección de la biodiversidad.

Ejemplo de lo anterior es la bioprospección, la cual cuenta con las bases para lograr un desarrollo sostenible en los países debido a la perspectiva ambiental, económica, política y cultural que maneja. Por consiguiente, la bioprospección debe ser entendida como una estrategia que permite adquirir nuevos conocimientos, mejorar la calidad de vida, brindar seguridad alimentaria, producir ganancias económicas, conservar la biodiversidad, evitar la erosión genética y respetar los derechos de las comunidades, permitiéndoles participar en la toma de decisiones y reconociendo su conocimiento tradicional. Este no se trata solo de los saberes y técnicas desarrolladas de generación en generación por una comunidad para la obtención de diversas variedades de vegetales, control de plagas o evitar la degradación del suelo, sino también se reconoce como alternativa de conservación de la naturaleza y es parte esencial de la cultura e identidad de las comunidades.

Adicionalmente, al regular el uso de las semillas para la siembra y resiembra, exigiendo el uso de semillas certificadas o prohibiendo la utilización de los conocimientos tradicionales agrícolas, se está viendo afectada la soberanía alimentaria del mundo, debido a que la variabilidad genética de los recursos se está disminuyendo, por ende, la variedad y resistencia de los productos agrícolas.

Actualmente, debido a la falta de claridad en las normas que aborda este contexto, la bioprospección no se ha podido desarrollar adecuadamente en el país, yendo en contravía con algunas leyes y acuerdos internacionales a los que Colombia está comprometido. Teniendo la necesidad que para poder llenar los vacíos legales que permiten esta irregularidad se desarrolle un tribunal ambiental, con personas competentes para entender desde lo jurídico, técnico y científico la compleja relación que existe entre los intereses económicos, sociales y de conservación de los recursos naturales y conocimientos tradicionales, así como los conflictos ambientales y de derechos de propiedad.

Debido a la falta de legislación adecuada e integral en los temas de acceso a los recursos genéticos y conocimientos tradicionales, sumado al fuerte interés económico de diferentes actores y Estados, estos conocimientos han sido utilizados sin el consentimiento de las comunidades campesinas y pueblos indígenas o sin darles el adecuado reconocimiento y la división justa y equitativa de sus beneficios, por lo cual se han buscado diferentes alternativas que permitan la adecuada protección de estas comunidades y de sus conocimientos tradicionales.

En este sentido, para proteger estos saberes es primordial que se les haga un reconocimiento desde la propiedad intelectual de las comunidades (su conocimiento tradicional), así como se ha venido desarrollando la protección de la biodiversidad. Colombia, como país megadiverso y agropecuario, debe liderar el desarrollo de normativas claras que integren todos los niveles de protección y que, a su vez, generen una ventaja competitiva en el mercado, con estrategias como la denominación de origen, entre otros. El objetivo es claro: seguir logrando un crecimiento económico y ser ejemplo de un Estado que respeta los derechos de las comunidades y la protección de los recursos naturales. 


\section{Referencias bibliográficas}

Acea-Valdés, Y. (2014). Realidad y derecho sobre los conocimientos tradicionales: especial referencia al sector agrícola en Cuba. Revista Novedades en Población, 10(19), 113121.

Álvarez-Tafur, M. (2014). La Decisión 391 de 1996 de la Comunidad Andina: su lectura antes y después del TLC de Colombia con Estados Unidos en materia de acceso y protección de los recursos genéticos y biológicos. Criterio Libre Jurídico, 21, 53-64.

Borrás, S. (2020). Bioprospección. Enciclopedia De Bioderecho Y Bioética. https:// enciclopedia-bioderecho.com/voces/47

Cafferatta, N. (2004). Introducción al derecho ambiental. Ciudad de México: Instituto Nacional de Ecología.

Carranza, X. (2017). La bioprospección de microorganismos en Colombia como uso sostenible de la biodiversidad. https://core.ac.uk/download/pdf/143454198.pdf

Comunidad Andina. (1996). Decisión 391 Régimen Común sobre Acceso a los Recursos Genéticos. https://www.wipo.int/edocs/lexdocs/laws/es/can/can011es.pdf

Comunidad Andina. (2003). Decisión 486 Régimen Común Sobre Propiedad Industrial. https://www.wipo.int/edocs/lexdocs/laws/es/can/can012es.pdf

Conabio-giz (2017). Conocimiento tradicional asociado a los recursos biológicos. Cuaderno de divulgación 1. Proyecto Gobernanza de la Biodiversidad: Participación justa y equitativa de los beneficios que se deriven del uso y manejo de la diversidad biológica, Ciudad de México.

Congreso de la República de Colombia. (1974). Ley 2811 de 1974. Diario Oficial 34.243. https://www.funcionpublica.gov.co/eva/gestornormativo/norma_pdf.php?i=1551

Congreso de la República de Colombia. (1991). Ley 21 de 1991. Diario Oficial 39720. http:// www.suin-juriscol.gov.co/viewDocument.asp?ruta=Leyes/1577376

Congreso de la República de Colombia. (1994). Ley 165 de 1994. Diario Oficial 41.589. https://www.minambiente.gov.co/images/BosquesBiodiversidadyServiciosEcosistemi cos/pdf/Politica-Nacional-de-Biodiversidad/3355_ley_0165_091194.pdf

Congreso de la República de Colombia. (2000). Ley 599 de 2000. Diario Oficial 44.097. http://www.secretariasenado.gov.co/senado/basedoc/ley_0599_2000.html

Congreso de la República de Colombia. (2002). Ley 740 de 2002. Diario Oficial 44.816. http://www.secretariasenado.gov.co/senado/basedoc/ley_0740_2002.html

Congreso de la República de Colombia. (2009). Ley 1333 de 2009. Diario Oficial 47.417. http://www.secretariasenado.gov.co/senado/basedoc/ley_1333_2009.html 
Congreso de la República de Colombia. (2011). Ley 1453 de 2011. Diario Oficial 48.110. http://www.secretariasenado.gov.co/senado/basedoc/ley_1453_2011.html

Congreso de la República de Colombia. (2018). Ley 1926 de 2018. Diario Oficial 50.664. http://www.secretariasenado.gov.co/senado/basedoc/ley_1926_2018.html

Congreso de la Republica de Colombia. (2020). Proyecto-Ley 047 de 2020. https://www. camara.gov.co/tribunales-ambientales

Consejo Nacional de Política Económica y Social. (2011). Documento compes 3697 de 2011. https://colaboracion.dnp.gov.co/CDT/Conpes/Econ\%C3\%B3micos/3697.pdf

Constitución Política de Colombia (1991). Gaceta Constitucional 116. http://www. secretariasenado.gov.co/senado/basedoc/constitucion_politica_1991.html

Corte Constitucional. (24 de junio de 1992). Sentencia T- 428 [M.P Ciro Angarita Baron]

Corte Constitucional. (03 de febrero de 1997). Sentencia SU-039 [M.P Antonio Barrera Carbonell]

Corte Constitucional. (10 de noviembre de 1998). Sentencia T- 652 [M.P Carlos Gaviria Díaz]

Corte Constitucional. (13 de mayo de 2003). Sentencia SU- 383 [M.P Alvaro Tafur Galvis]

Corte Constitucional. (26 de octubre de 2006). Sentencia T-880 [M.P Alvaro Tafur Galvis]

Corte Constitucional. (23 de enero de 2008). Sentencia C-030 [M.P Rodrigo Escobar Gil]

Corte Constitucional. (14 de mayo de 2008). Sentencia C-461 [M.P Manuel José Cepeda Espinosa]

Corte Constitucional. (10 de octubre de 2009). Sentencia C- 715 [M.P Gabriel Eduardo Mendoza Martelo]

Corte Constitucional. (29 de octubre de 2009). Sentencia T-769 [M.P Nilson Pinilla Pinilla]

Cotes, A., Barrero, L., Rodríguez, F., Zuluaga, M. y Arévalo, H. (Eds.). (2012). Bioprospección para el desarrollo del sector agropecuario de Colombia. Corporación Colombiana de Investigación Agropecuaria.

Duarte, O. y Velho, L. (2009). La bioprospección como un mecanismo de cooperación internacional para fortalecimiento de capacidades en ciencia y tecnología en Colombia. Ciencia Da Informaçao, 38(3), 96-110. https://doi.org/10.1590/S010019652009000300007

Getgen, J. (2006). Bioprospecting and Indigenous Cultural Rights: Creating Authorship, Agency, Empowerment and the Next Generation of Biopirates? SSRN Working Paper. http://dx.doi.org/10.2139/ssrn.1722710 
Grau, J. (2013). Convenio sobre la Diversidad Biológica: la última oportunidad de evitar la tragedia, acorralada. Ecología Política, 46, 25-35. Helfer; L.R. (2002). Derechos de propiedad intelectual sobre variedades vegetales. Una Visión de conjunto con opciones para los gobiernos nacionales. Estudio legislativo de la FAO, 31. Recuperado de http://www.fao.org/3/a-bb064s.pdf

Hernández, A. (2000). Biodiversidad y variedades vegetales. La protección de las nuevas variedades vegetales y su relación con la diversidad biológica. Bogotá: Instituto de Investigación de Recursos Biológicos Alexander von Humboldt.

Instituto Colombiano Agropecuario. (2010). Resolución 970 DE 2010. Diario Oficial 47.648. http://www.avancejuridico.com/actualidad/documentosoficiales/2010/47648/r ica_0970_2010.html

Instituto Colombiano Agropecuario. (2015). Resolución 3168 DE 2015. Diario Oficial 49.632. http://www.suin-juriscol.gov.co/viewDocument.asp?ruta=Resolucion/30033935

Matiz, C., Rodríguez, G. y Zuluaga, G. (2007). Flora medicinal y sus conocimientos asociados. Lineamientos para una reglamentación. Universidad del Rosario.

Melgarejo, L., Sánchez, J., Chaparro, A., Newmark, F., Santos, M., Burbano, C. y Reyes, C. (2002). Aproximación al estado actual de la bioprospección en Colombia. Universidad Nacional de Colombia.

Mendoza-Cerquera, N. (2015). De los conocimientos ancestrales indígenas: una ruptura entre lo propio y lo ajeno. Documento de trabajo de la Línea de Investigación en Derecho Ambiental de la Universidad del Rosario.

Ministerio de Agricultura y Desarrollo Rural. (2006) Resolución 187 de 2006. Diario Oficial 46.356. https://www.ica.gov.co/getattachment/efc964b6-2ad3-4428-aad5a9f2de5629d3/187.aspx

Ministerio de Ambiente y Desarrollo Sostenible. (2014). Resolución 1348 de 2014. Diario Oficial 49.259.https://www.icbf.gov.co/cargues/avance/docs/resolucion_ minambienteds_1348_2014.htm

O'connor, J. (1998) Natural Causes: Essays in Ecological Marxism. Guilford Press.

Organización Mundial de la Propiedad Intelectual (ompi) (2002). Comité Intergubernamental sobre Propiedad Intelectual y Recursos Genéticos, Conocimientos Tradicionales y Folclore. Tercera sesión. Ginebra: WIPO/GRTKF/IC/3/9.

Organización Mundial de la Propiedad Intelectual (OMPI). (2005). Propiedad intelectual y conocimientos tradicionales. Publicación de la OMPI 920(S). Recuperado de http:// www.wipo.int/edocs/pubdocs/es/tk/920/wipo_pub_920.pdf 
Organización de las Naciones Unidas (2007). Declaración de las Naciones Unidas sobre los derechos de los pueblos indígenas.

Organización de las Naciones Unidas para la Agricultura y la Alimentación [FAO]. (2009). Tratado Internacional sobre los recursos fitogenéticos para la alimentación y agricultura.http://www.fao.org/pgrfa-gpa-archive/hnd/files/Tratado_internacional_ sobre_los_recursos_fitogeneticos_para_la_al imentacion_y_la_agricultura.pdf

Organización de las Naciones Unidas para la Agricultura y la Alimentación [FAO]. (1996). Cumbre Mundial sobre la Alimentación. http://www.fao.org/wfs/index_es.htm

Organización de las Naciones Unidas para la Educación, la Ciencia y la Cultura [UNESCO]. (201). Declaración Universal sobre la Diversidad Cultural

Organización Mundial del Comercio [OMC]. (1994). Acuerdo sobre los Aspectos de los Derechos de Propiedad Intelectual relacionados con el Comercio. https://www.wto. org/spanish/tratop_s/trips_s/trips_s.htm

Pino Miklavec, N. (2013). Tutela judicial administrativa de los intereses ambientales: estudio comparativo de los ordenamientos español y argentino. Madrid: Aranzadi.

PNUD (2010). Incorporación del conocimiento tradicional asociado a la agrobiodiversidad en agro-ecosistemas colombianos. Recuperado de http://www.co.undp.org/content/ colombia/es/home/ourwork/crisispreventionandrecovery/news/incorporacion-delconocimiento-tradicional-asociado-a-la-agrobio.html

Posey, D., y Dutfield, G. (1996). Beyond Intellectual Property. Toward Traditional Resource Rights for Indigenous Peoples and Local Communities. Ottawa: International Development Research Centre.

Presidencia de la República. (1996). Decreto 1397 de 1996. http://www.suin-juriscol.gov.co/ viewDocument.asp?ruta $=$ Decretos $/ 1279607$

Presidencia de la Republica (2005). DECRETO 4525 DE 2005. http://www.suin-juriscol.gov. co/viewDocument.asp?id $=1547044$

Presidencia de la República. (2015). Decreto 1076 de 2015. https://www.funcionpublica.gov.co/eva/gestornormativo/norma.php?i=78153

Procuraduría General de la Nación (2009). El derecho al territorio: garantía para la pervivencia de los grupos étnicos. Bogotá, PGN.

Programa de las Naciones Unidas para el Desarrollo (2020). Programa de las Naciones Unidas para el Desarrollo. https://www.undp.org/content/undp/es/home/ sustainable- development-goals.html 
República de Colombia, Contraloría General de la Republica (2012). Informe del estado de los recursos naturales y del ambiente 2011-2012. Bogotá: Imprenta Nacional.

República de Colombia, Ministerio de Ambiente y Desarrollo Sostenible (2012). Política Nacional para la Gestión integral de la Biodiversidad y sus Servicios Ecosistémicos | Ministerio de Ambiente y Desarrollo Sostenible.

Rodríguez, G. A., Gómez Rey, A., \& Sáenz Quitian, A. (2014). Conflictos entre propiedad, comercio y ambiente. Alejandra Sáenz Quitian, Andrés Gómez Rey, Gloria Amparo Rodríguez, editores académicos. Ibañez.

Rodríguez, G. y Vargas-Chaves, I. (2016). La prevención en materia ambiental: tendencias actuales: Material de estudio. Universidad del Rosario.

Sunder, M. (2007). The invention of traditional knowledge. Law and contemporary problems, $70(2), 97-124$.

Vargas-Chaves, I. (2018) La bioprospección y el rol de la consulta previa en la prevención del daño sobre los conocimientos tradicionales. En M. Lerner-Wodnicky, M. Urueña y

I. Vargas-Chaves. (Eds.) Entre libertad e identidad: debates comercio-cultura desde una aproximación Latinoamericana. (pp. 71-88). Universidad La Gran Colombia.

Vargas-Chaves, I., Rodríguez, G. y Blumenkranc, H. (2020). Propiedad Intelectual sobre los conocimientos tradicionales agrícolas. Elementos para su caracterización en el ordenamiento jurídico colombiano. Universidad del Rosario.

Von Lewinski, S.(2008). Indigenous heritage and intellectual property: genetic resources, traditional knowledge and folklore. Kluwer Law International BV.

Williams, T. y Hardison, P. (2013). Culture, law, risk and governance: contexts of traditional knowledge in climate change adaptation. En J. Koppel (Ed.). Climate Change and Indigenous Peoples in the United States. (pp. 23-36). Springer.

Wynne, B. (1992). Uncertainty and environmental learning: reconceiving science and policy in the preventive paradigm. Global Environmental Change, 2(2), 111-127. 